\title{
Methodology of Deep Perception of a Person's Mind
}

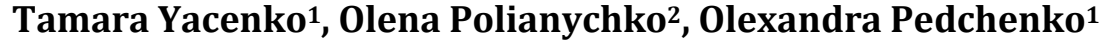 \\ ${ }^{1}$ Department of Deep Correction and Psycho-Social Rehabilitation, The Bohdan Khmelnytsky National \\ University of Cherkasy, Cherkasy, Ukraine \\ ${ }^{2}$ Department of Physical Education, Borys Grinchenko Kyiv University, Kyiv, Ukraine \\ Email: ts_yacenko@mail.ru,pony2006@ua.fm
}

Received 5 May 2016; accepted 12 June 2016; published 15 June 2016

Copyright @ 2016 by authors and Scientific Research Publishing Inc.

This work is licensed under the Creative Commons Attribution International License (CC BY).

http://creativecommons.org/licenses/by/4.0/

(c) (i) Open Access

\begin{abstract}
In this article, an issue is raised on methodology of forming competency in a future psychologist on the basis of adequate understanding of wholeness of the psychic; comparison of academic and psychodynamic approaches to the problem of academic training of a psychologist is done as well as analysis of the leading sections in psychology relevant to psychodynamic approach.
\end{abstract}

\section{Keywords}

Active Social-Psychological Perception (ASPP), Probabilistic Forecasting, Deep Correction, Deep Perception, Methodology

\section{Background/Objectives and Goals}

The way of forming competency in a future psychologist cannot stay out of methodology, which involves not only mastering of theoretical knowledge by a future psychologist but also the practice of diagnostic and correction process in groups of Active Social-Psychological Perception (ASPP).

It has been established that a theory is a set of beliefs, ideas aimed at the interpretation of certain events; the highest organization form of knowledge promotes a holistic view of a certain area of reality in its comprehensive interconnections. Methodology is a system of principles and methods of organization and construction of theoretical and practical activities, and a theory of this system. Methodology is based on a system of proven rules and norms of perception (and their relevant actions) that best relate to the properties and laws of reality.

Psychodynamic paradigm aligns attention with the joint progress to the truth in an active interaction of a psychologist with a respondent (participant in the process of deep perception of the psyche). Ethical appropriateness of perception acquires the significance in the context of perception of the psyche in its integrity (con- 
scious/unconscious). The purpose is defined only in general, like a guide of a psychodynamic process that is clarified in the dynamics of semantic appropriateness of the subject activity, progress of which becomes consistent with the objective logic of perception.

These are positions that underlie psychodynamic approach to the training of a psychologist, overcoming a simplistic mechanistic view of the understanding of the subject of psychology. Psychodynamic theory (due to positive results of diagnostic and correction practices over years) evidenced that the psyche is whole in the presence of functional differences and autonomy of the conscious and unconscious spheres. Psychodynamic approach is based on the following: a holographic structure of the unconscious (thereby "psyche knows everything" and at the same time "it does not know what it knows"); involuntary activity of a subject (in which some elements of the psychic embody the essence of the whole), procedural diagnostics, which is the basis of deep perception.

\section{Methods}

Empirical material persuades of the existence of internal functional arrangement of the psychic that has relevance to information equivalents, which combine both spheres of the psyche. The article states the continuity of information exchange between subsystems (conscious/unconscious), including the transfer of ideal (spiritual) reality into materialized not only into physiological ("brain”), but also into external subjectified, visualised one, which opens perspectives of deep perception according to laws of the conscious to a psychologist. That is why the process of deep perception in ASPP groups (groups of active social-psychological perception) is invariably mediated by presenter (sculpturing, drawing, stone models, etc.) created by its participants.

\section{Expected Results/Conclusion/Contribution}

Deep psychologist relies on the assumption of unity of the conscious and the unconscious, which focuses on the horizon of their contact, and not the line of their separation. The entire procedure of active social-psychological perception is aimed at objectification and restoration of damaged interconnections (information exchange) between substructures of the psyche that finds expression in subjectivism (distortion) of displaying activity of an individual and leads to social failures, maladaptation under pressure of consistence of objective reality. Subjectivism of psychic image is caused by objective (though ideal) reality of laws of functioning of the unconscious (i.e., other sphere), which manifests itself in the invariance of psychic characteristics generated by the defensive system. The latter give rise to the effect of possible predictability of behaviour by external observer (psychologist).

Humanistic organizational principles of the process of active social-psychological perception contribute to levelling of contextual (peripheral) forms of psychological defence, opening the prospect of approaching the perception of basal forms of defence based on implied core values. Given the deep self-perception, a future expert surveys diagnostic and correction process in the "probabilistic forecasting" oriented to perception of behavioural material logical ordering. The latter facilitates detection of inconsistency of energy direction of the "logic of the conscious" and the "logic of the unconscious" ("other logic") in the ASPP process.

An important step in professional development of a future psychologist is mastering the category of "probabilistic logic", which includes interim true value (the probability of the truth of statements), which is the base for forecasting their further proof by empirical material. If the term "probability" is associated with a particular event, act, which finds empirical evidence, then "reliable logic" is elaboration of "inductive logic". In this case, there is transfer from the language of forecasts (under the influence of statements (behaviour) of a respondent) to their confirmation by revealing the ordering (consistency) of behavioural material obtained in dialogic interaction. The specificity of reliable logic of deep perception is due to the lack of capacity of complete elimination (reduction) of the probability. In other words, a factor of incomplete probability is invariably present in deep perception, with dynamics up to clarification of meanings of behavioural acts, in the direction from the field of the significance to the uniqueness.

Researches in psychodynamic format confirm the fact that the probabilistic logic is a kind of multiple-valued logic that is not limited to two-valued property ("true or false") only. The process of deep perception takes into account the availability of symbolism and its polyvaluedness. The latter implies the probability of the truth through the lack of a priori certainty of content (meaning) of spontaneous behaviour that can be overcome by batch supply of information to a respondent by a psychologist. Spontaneity of behaviour of one or another re- 
spondent is distinguished with the frequency of manifestations of iterative, invariant, repetitive behaviour patterns, having proximity to the concept of relative frequency by its content. Researches are also directed to an issue of statics and dynamics of organization of the psychical in the integrity of its functioning. In this context, the role is played by concepts such as disposition, setting, tendency, a dynamic stereotype that contribute to defining the vector of direction of an individual's energy, depending on "mortido" or "libido".

So, probabilistic forecasting underlying deep perception facilitates to revealing the internal "need" that makes its way through a set of coincidences in their multiplicity and polyvaluedness of symbols. Specification of "need” is predetermined by logical ordering of the psyche, which is narrowed by a process of interpreting of the associative interconnections in behavioural material (dynamics from symbolic polyvaluedness to significance). In such circumstances, the factor of spontaneous actions will be effective until its underlying causes get revealed. Deep psychological perception introduces changes to circumstances of inner world of a future psychological expert exclusively by revealing the true significance of a visualized presenter (meaning), as well as its impact on energy direction of the psyche. The latter occurs by obtaining the reflective knowledge by a perception person, dispelling their own illusions generated by defences and deepening the understanding of infantile determinants of personal issues (internal contradictions). All this contributes to the reconstruction of primitive structures of the psyche through multiple-level expansion of a person's self-consciousness.

Logical or inductive probability characterizes the relationships between a message (foundation, postulate) and conclusions of inductive thinking. Unlike deduction, initial inductive positions do not guarantee true conclusions, but merely make them probabilistic, that is believable. Under such circumstances, the probability expresses not so much a degree of subjectivity, as reveals a "reasonable belief" predictability of a desired result.

Activity of the ASPP team leader is based on probabilistic assumptions of logical ordering of behavioural material, and rational-logical interpretations of diagnostic and correction process relate to an analysis of features of perception of spontaneous ("subjective”) person's activity. The ability to prevent algorithmization of acts of a deep correction process leader is an integral part of both a general methodical strategy of the ASPP method and its partial methodologies, which are adjusted to current diagnostic and correction issues in the "here and now" situation. The latter corresponds to theoretical and methodological foundations of deep perception as well as a psychodynamic model of optimal diagnostic and correction, analytical and dialogue work of a psychologist with a respondent.

General personal changes in a future psychologist have been already set by group principles of work arrangement in ASPP groups: acceptance of other people (and yourselves) as they are; sincerity; an analysis of behaviour of other people (and self-analysis); a lack of criticism (and self-criticism); formation of skills "to be in the situation and be over it"; decentration of attention; overcoming the "tunnel vision" effect; transforming yourselves into a "research subject"; weakening both tendencies to own "psychic importing" and tendencies "towards psychological death" and so on.

Individual personal changes: dynamics of individualized development is catalysed by a process of positive disintegration and secondary integration of the psyche at the higher level of development, due to revealing its internal ordering detection and resolution of personal issues (inner contradictions) by a future psychologist, leading to harmonization of his/her psyche.

Personal professional changes correspond to general psychodynamic line of creative selection of working methods and tools to meet the needs of a deep process of "here and now" according to the individual psychical uniqueness of a particular person. The ASPP process encourages the development of empathy, sensitivity, intuition, self-analysis skills (self-reflection) and longitudinal analysis (interpretation) of behaviour of other people. Development of abilities to procedural psychical diagnosis in its unity with correction takes place in a future psychologist during studies.

Personal growth generalized indicators of future applied psychologists as a result of ASPP groups: openness to new experiences; attention to acts of spontaneous behaviour of another person; prevalence of objectivity in assessing the conduct of another person on projective-emotional (subjectivized) assessment; social activity, adequacy and adaptability; weakening of stereotypes and automation of behaviour; reduction in depressive and regressive tendencies of behaviour; ability to reveal their own constructive potential, which implies reducing the mortido (destructive) energy and expanding the creativity horizons in the form of professional self-realization, etc.

The main advantage of psychodynamic approach to revealing the theoretical and methodological and methodical foundations of deep perception of the psyche by a future expert is his/her focus on universal laws of 
nature of the psychical that allows him/her to find methodological support in understanding of general and individualized aspects of the psyche. Own internal motivation of a future psychological for self-perception (as well as the choice of profession), which is a powerful catalyst for optimization of his/her personal characteristics, is important.

Researches convince of the need to ensure a holistic approach to improvement of the training of applied psychologists. The psychodynamic theory integrates seamlessly into the general training programme for psychologists, expands the horizons of personal growth, development of reflective intelligence and sensitivity of a future expert. All this greatly complements a format of academic training of psychologists in its orientation to mastering the standard knowledge. Moreover, the passage of deep correction catalyses personal changes towards the harmonization of the psyche structure, development of those characteristics of a person that are desirable for the formation of professional excellence and ensuring the effectiveness of psychological counselling.

Progressive philosophic views, which imply the results of various sciences, help in the study of professional and personal issues in the training of future psychologists. The psychodynamic approach, which has been developed over past thirty-five years, has influenced the content of psychologists' training at universities and the Psychological Service of Ukraine. However, methods, which take into account possibilities of perception of the psychic in its integrity on the basis of reflexive knowledge, have been still not enough implemented in the training process.

The main task of the perception theory is connected to revealing the dynamics of this process. Psychodynamic paradigm has gained demarcation of academic and experimental psychology. We associate ourselves with K. Lewin, who noted that "experimental psychology generates semi-pattern" [1]. By perceiving the sphere of the conscious only, the person's inner world will be perceived and studied only halfway, thus, academic search for the truth of revealing the nature of the psyche within classical determinism does not provide an adequate solution to the issue of perception of the psychic.

Determinism (from Latin determine-I define) is a philosophical doctrine of objective, logical interconnection and interdependence of phenomena of the material and the spiritual world. The essential feature of determinism is causality that determines connections between phenomena where the cause (under sufficiently certain conditions) with the need creates another phenomenon like after-effect. Of course, there is no phenomenon beyond causality. At the same time, the psychic cannot be attributed to one-causal phenomena. Considering the methodological positions of professional training of a psychologist, we should note that perception subject is the holistic psyche in its conscious and unconscious aspects. With this in mind, we cannot perceive the psychic in its integrity with a limited focus on perception cause-and-effect aspects of the conscious, where the unconscious can be never directly represented. Famous Georgian scholar A.E. Sherozia indicates: “... from the very beginning of its development psychology let us know that it is incapable to reproduce a holistic picture of the psyche, relying on one Galilean principle only" [2]. We hold to the position that, like quantum physics, we need to introduce a complementarity principle into the research space for perception and adequate understanding of the psychic. Given the fact that the complementarity principle has been introduced by N. Bohr [3] in quantum physics with the aim of perception of quantum and Newtonian circumstances of the world in their unity, A.E. Sherozia notes: “... in some way, the complementarity principle connects two large and equally fundamental sciences of the world: physics as a science of physical (trans-psychic) reality (broadly defined) and psychology as a science of psychic reality (similar broadly defined), linking them so that one and another sciences complement each other in a holistic scientific picture of the world" [2]. N. Bohr clearly pointed to "the need to replace the classical ideal of causality to the complementarity principle (simplified-complementarity)” [3].

Said above principle relates to those objects which exhibit asymmetry of functions. In the case of the psychic, such asymmetry is functionally inherent to the spheres of the conscious and the unconscious, making the rectilinear determinacy perception of connections between them impossible. Connection is done through certain information equivalents (in the noncompliance of functions and direction of energy potential). So, complementarity concerns establishment of connections between the sphere of the conscious and the other sphere (the unconscious). More accurately, we are referring to certain logical compatibility, balance. Information equivalents connect contradictory aspects (the conscious-unconscious) of the same reality - the psychic. Therefore, "psychology, just like quantum physics, should use the complementarity principle as a method of reproduction and perception of the integral psyche” [2].

Due to the fact that the unconscious represents the "objective reality" (due to its unconsciousness, and therefore no intervention of the conscious) it could be perceived as reality in general through the conscious. S. Freud 
pointed out that "all our knowledge is always connected with the conscious: we can even perceive the unconscious only by its transformation into the conscious" [4]. The unanimous opinion is that the unconscious in its directly unobservable form is not subject to rectilinear consciousness (perception). However, just as direct access is closed for the consciousness to the other sphere, so vice versa the unconscious cannot be rectilinearly explicated, and this encourages looking for a third way associated with the mediation, which creates visualization of the psyche, and dialogic interaction with a respondent. The latter corresponds to the guidelines of S. Freud: “... in order to bring the unconscious to the conscious, you need to create mediated chains first. Internal processes become perceptions through the verbal mediation" [4].

The above material shows that the training of future psychologists requires attention to the methodology of perception of the psyche in its integrity (conscious/unconscious). The research proves that perception of the unconscious cannot be achieved without attention to dynamic and structural aspects of the psychic (superego, I and It). A process of repression plays an important part in the formation of psychic substructures. However, the unconscious not only consists of the repression processes, but it is just repressed itself that turns it into the unperceived and gains dynamics during calling its like. In perception of integrity of the psyche, we believe that our knowledge is constantly connected to the conscious that is why we can perceive the unconscious only by one way, which is turning it into the conscious. The central methodological position lies in this moment and requires methodical and instrumental art of deep perception related to the objectification of information equivalents. The latter means the presence of something third (i.e. a mediator) between the spheres of the "conscious/unconscious". A remark by Freud of a way of psychoanalytic perception "through a combination of relevant verbal representations" [4] is important. With this in mind, a visualized presenter gains a mediation function only through its "revitalization" in dialogic interaction of a psychologist with a respondent.

S. Freud stressed the importance of actualization of "visual intelligence", especially in the practice of psychoanalysis of dreams. In this case, relationships between images, which require perception, are left beyond visual intelligence. Given the figurativeness of language of the unconscious imagery, the ability of symbols to embody the more synthesized content of the other (unconscious) becomes understandable, and this involves their research purposiveness of using the visualized material (toys, stone models, drawings, sculpturing, etc.) in deep perception. However, visual images are not enough for perception of the unconscious, it is necessary to identify interconnections between symbols in their many-valuedness and verbal aspects of thinking. It is a moment that is considered when a psychologist gives an impulse (stimulus) of visualization, self-introduction of a person, providing the next step, which is dialogic interaction with a respondent. Our practice over many years has proved the possibility and unconditional effectiveness of introduction of mediations in the study of latent aspects of the psychic and its study in the "observable plane".

So here are the conclusions: deep perception should have a mediated nature; visualization of primary "mediation" is desirable; materialized fixation of a "mediator" serves as an objective benchmark of perception; secondary mediation requires "revitalization" of self-presenter in the dialogic interaction (psychologist respondent); a process of dialogic interaction is carried out in a step-by-step, multi-level manner, with adherence to the rules of positive disintegration of the psychic and its integration at the higher level (through partial disintegration and integration); psychologist's questions are oriented to the internal implied ordering of the psychic, which is in synthesis with archetypal essence of the psychic; diagnostic capacity of questions (in terms of compliance with the internal ordering) generated an impulse in a person, a flow of energy that manifests itself in a person's motivation to both self-perception and the activity as a whole.

Summarizing, we would like to note that dialogic interaction of a psychologist with respondent gains mediation between two spheres of the psychic given its consistency with the internal ordering of the psyche. A dialogue, following the phenomenon of the psychic (spontaneous activity of a respondent) has no direct relationship to one or to another spheres of the psyche, it is focused on the horizon of their contact. Work is carried out not with individual components of the psyche, but with a person, the activity of whom is caused by motivation and self-improvement. A dialogue is not aimed at establishing the personal relationships, but at the information exchange, all of which contribute to the interpretation and objectification of information equivalents potentially relevant to the psychic in its integrity.

Psychodynamic paradigm empirically confirms the fact of holistic essence of the psychic (ideal) and its ability of presentation in observed (visualized) presenters, undergoing their conscious comprehension. Information equivalents combine the conscious and the unconscious, which is not within eyeshot in itself. This is because of the following: a) the unconscious never objectifies its content rectilinearly; b) a presenter of the "other" (ideal) 
in objectified, static form cannot convey the meaning of a living instance with emotive potential. Such specific format view of deep perception is stated by a classic thesis: "any knowledge comes from external perception", which has been taken as a basis for behavioural direction. Everything from the outside has a prospect to gain a note of the truth.

Pretty often, adequate perception of the psychic in its integrity is limited by an academic approach, in particular, the desire of mathematical evidence of phenomena. Hegel pointed to the one-sidedness of this approach: "Mathematization gives the matter only the properties of space-time" (quote from [5]). Conclusion: mathematization pushes into the darkness the perception of the other, timeless and spaceless sphere, which is the unconscious. ASPP dialogue-based deep perception takes into account continuum of manifestation of internal system organization of the psychic which is possible in the event of dialogue following a "respondent", semantics of his/her language symbolism. By the way, I. Kant condemned that science which does not enter into a dialogue with nature, and imposes its language to the latter. In productive dialogic interaction, a single act of behaviour (question-answer), with a code already present, becomes important, given the holographic essence of the other (unconscious) sphere-the partial, single "speaks" about the characteristics of the whole, that is available for perception in ASPP groups.

It is interesting that S. Freud has also used the "other" term compared with the conscious, indicating that it integrates repression [4]. We find the common in the understanding of the mediation issue that cannot directly touch the Id (as a reservoir of energy of instincts), it is inaccessible to verbal stimulation. Verbum may correlate only with that sphere that was formed as a result of unrealized Id instincts through the action of the Superego censorship. This is one aspect of the formation of the preconsciousness, which is in the field of our research attention.

Against the background of transparency of the abovementioned thoughts that unite us with psychoanalysis, there is a problem left in the dark: what exactly aspects of the unconscious will be drifted "at the surface" in case of a spontaneous behaviour of a respondent?

Let us try to explain. Experiences (feelings) facilitate the transition of the internal into the external. The satisfaction-dissatisfaction series serves as an energy stimulus. Experiences that appropriate to the satisfaction principle lead to relaxation, while dissatisfaction retains the potential of motivation for activity. Conventionally, satisfaction can be designated as reduced energy, dissatisfaction as increased energy, "begging" to the implementation in action. It is not for nothing S. Freud said that "we draw energy for creativity in unsatisfied needs".

Id foci repressed through the synthesis and integration with the priority of emotive trace dominance, influence the formation of such a phenomenon as "beyond the experiential", which explains the preservation of energy potential of person's unsatisfied needs. Therefore, the objectification of the unconscious in deep perception is accompanied by revitalization of negative and sometimes dramatic trace effects that find their symbolic and figurative expression in the process of creating the visualized presenters (drawings, stones models, sculpturing).

The psychodynamic approach, pointing to integrity of the psychic, is far from perception thereof as "additive sum". We are dealing with holographic integrity, and this is true integrity, which does not occur by means of "arithmetic" - a sum of its parts, and provides high-quality, system integration thereof. The unconscious can only be included in dynamic integrity, which is reflected in each partial manifestation. Deep perception is aimed at revealing the meanings of these "manifestations" by their deployment in the temporal (time) characteristics.

\section{Conclusions}

Deep perception requires the consideration of the following:

- Specificity of functional interconnections in the integral psyche (conscious/unconscious);

- Energy activity of the sphere of the unconscious, manifestation of which is possible in minimizing (levelling) the external stimuli of the ASPP participants' activities in the situation of "here and now";

- Archetypal psychic capacity to self-objectification (sculpturing, drawings, etc.), with recoding the latent meanings into visualized form;

- Mediation "mission" of a visualized presenter between the external and internal aspects of the psychic (conscious/unconscious);

- Nature of dialogic interaction of a psychologist with a respondent that promotes emotional "revitalization" of a presenter as a prerequisite for its transformation into a mediator;

- Need to focus on the significance of the events experienced by a respondent shown in trace effects that de- 
fine the impulse of responses of a respondent to question of a psychologist;

- Differences between two aspects of psychic reality (conscious/unconscious): their autonomy and inexistence apart from each other;

- Need for probabilistic forecasting based on probability of interconnections, mutual transitions from the energy (unconscious) sphere into the semantically understandable as a result of interpretation (explanation);

- Information privacy of presenters, which stipulates the need for their decoding in dialogic interaction of a psychologist with a respondent.

Research perspectives are seen by us in the more functionally capacious and reliable detection of the "information equivalents" category as a form of information "merging" of two spheres of the integral psychic.

\section{References}

[1] Lewin, K. (2001) Field Theory in Social Science. Dynamic Psychology: Selected Papers. Smysl, 251-270.

[2] Sherozia, A.E. (1978) Dialectics, Complementarity Principle и Psychological Integrity Perception Problem: To NonClassically Oriented Strategy of Scientific Experiment in Psychology. In: Bassin, F.V., Prangishvili, S. and Sherozia, A.E., Eds., The Unconscious, Vol. 3, Metsniereba, Tbilisi, 751-788.

[3] Bohr, N. (1971) Causality and Complementarity. Selected Research Papers, In: Tamm, I.E., Fock, V.A. and Kuznetsov, V.G., Vol. 2, Nauka, 204-212.

[4] Freud, S. (1990) I and It. Mottem, 55 p.

[5] Prigogine, I. (2005) Order out of Chaos: Man’s New Dialogue with Nature. Translated from English by Y.A. Danilova, General Editorship and Afterword: V.I. Archinov, Y.L. Klimontovich, and Y.V. Sachkov, 5th Edition, Kom Knigaa, 294 p. 